\title{
Assessment of two alternative sample transport and fixation methods in the microbiological diagnosis of bacterial vaginosis
}

\author{
Erica Eason SM MDCM FRCSC 1,3 , Baldwin Toye MD FRCPC ${ }^{2,4}$, \\ George A Wells MSc PhD ${ }^{3}$, Mary Senterman MD FRCSC FRCPC ${ }^{1,2}$
}

E Eason, B Toye, GA Wells, M Senterman. Assessment of two alternative sample transport and fixation methods in the microbiological diagnosis of bacterial vaginosis. J Sex Reprod Med 2003;3(4):133-137.

BACKGROUND: The standard method for specimen collection and transport for microbiological diagnosis of bacterial vaginosis is an air-dried smear of vaginal secretions, promptly heat- or alcohol-fixed, Gram-stained and scored by Nugent's criteria.

OBJECTIVE: Two alternative methods are evaluated: sending a swab in transport medium to be smeared and Gram-stained in the laboratory two days later; and sending a smear of vaginal secretions sprayed with cytological fixative to the laboratory for Gram staining seven days later.

PATIENTS AND METHODS: One hundred fifty-two women aged 18 years and older who attended a hospital colposcopy clinic or a community healthy sexuality clinic were studied. This was a prospective study: three vaginal swabs were taken from each patient and handled as described above. Each slide was blindly and independently interpreted by two microbiology technologists. The sensitivity, specificity and coefficient of agreement of the transported swab and cytologically fixed methods were compared with the air-dried smear method.

RESULTS: Smears from swabs in transport medium and cytologically fixed smears both had 90\% sensitivity and 97\% specificity for bacterial vaginosis compared with diagnosis from air-dried smears. Cohen's kappa was 0.88 (95\% CI 0.79 to 0.97 ) for each method. Inter-rater reliability assessed over all slides (all sampling techniques) was excellent (kappa 0.94).

CONCLUSIONS: For the diagnosis of bacterial vaginosis, both alternative techniques provide results equivalent to air-dried direct smears. A vaginal smear sprayed with cytological fixative provides immediate fixation of material to the slide, permits delays in swab transport and avoids the requirement for transport at a controlled temperature imposed by swabs.

Key Words: Bacterial vaginosis; Sampling techniques
Méthode de fixation et de transport des prélèvements pour un diagnostic microbiologique de vaginose bactérienne : évaluation de deux variantes

CONTEXTE : La méthode classique de prélèvement et de transport des échantillons pour un diagnostic microbiologique de vaginose bactérienne comprend quatre étapes : le séchage à l'air des frottis de sécrétions vaginales, la fixation rapide à la chaleur ou à l'alcool, la coloration de Gram, puis l'examen selon les critères de Nugent.

OBJECTIF : Évaluer deux variantes de la méthode classique : envoyer des écouvillons dans un milieu de transport en vue d'un frottis et d'une coloration de Gram, deux jours plus tard au laboratoire; envoyer des frottis de sécrétions vaginales, traités par l'application d'un fixatif cytologique pulvérisé, sept jours plus tard au laboratoire en vue d'une coloration de Gram.

PATIENTES ET MÉTHODE : Cent cinquante-deux femmes âgées de 18 ans et plus, qui se sont présentées à une clinique de colposcopie en milieu hospitalier ou à une clinique communautaire de santé sexuelle ont participé à l'étude. Il s'agissait d'une étude prospective : trois prélèvements vaginaux ont été effectués chez toutes les patientes et ceux-ci ont été traités selon les méthodes décrites précédemment. Chaque frottis a été interprété en aveugle et de façon indépendante par deux technologistes en microbiologie. La sensibilité, la spécificité et le coefficient de concordance des méthodes de transport des écouvillons et de fixation cytologique ont été comparés à la méthode de séchage des frottis à l'air.

RÉSULTATS : Les frottis effectués à partir des écouvillons conservés dans un milieu de transport et les frottis traités par le fixatif cytologique ont produit une sensibilité de $90 \%$ et une spécificité de $97 \%$ par rapport aux frottis séchés à l'air pour le diagnostic de vaginose bactérienne. Le test de concordance de Cohen a atteint 0,88 (IC à $95 \%$ : 0,79-0,97) pour chaque méthode. Le coefficient d'objectivité pour l'ensemble des frottis, traités selon les trois techniques, a été excellent (concordance : 0,94).

CONCLUSIONS : Les deux variantes ont donné, pour le diagnostic de vaginose bactérienne, des résultats équivalents à ceux produits par la technique classique du séchage des frottis à l'air direct. Toutefois, la pulvérisation d'un fixatif cytologique sur les frottis vaginaux provoque une fixation immédiate des prélèvements, ce qui permet d'en retarder le transport et d'éviter les contraintes liées au transport d'écouvillons sous température réglée.
Clinical diagnosis of bacterial vaginosis is based on Amsel Cet al's (1) criteria: homogenous watery discharge, amine odour with application of $10 \%$ potassium hydroxide, $\mathrm{pH}$ greater than 4.5 and clue cells on a wet mount of vaginal discharge. Clinical diagnosis without laboratory confirmation is accepted or even optimal $(2,3)$. However, it requires a good microscope at hand, microscopy expertise, reagents, time and tolerance of the unpleasant odour produced; it is also subjective (3). In actual practice, laboratory diagnosis is widely used instead, because it is easier and more convenient for the

\footnotetext{
${ }^{1}$ Department of Obstetrics and Gynaecology; ${ }^{2}$ Department of Pathology and Laboratory Medicine; ${ }^{3}$ Department of Epidemiology and Community Medicine; ${ }^{4}$ Department of Medicine, University of Ottawa, Ottawa, Ontario

Correspondence: Dr Erica Eason, Ottawa Hospital, General Campus, Box 803, 501 Smyth Road, Ottawa, Ontario K1H $8 L 6$.

Telephone 613-737-8683, fax 613-739-6266, e-mail eeason@ohri.ca

Received for publication July 2, 2003. Accepted September 29, 2003

Originally published in The Canadian Journal of Infectious Diseases 2003;14(6):322-326
} 
clinician (4) and provides an objective, reproducible result. The standard procedure for the microbiological test is to prepare a direct smear of vaginal discharge, air-dry the smear, and send it to a laboratory for heat or alcohol fixation, Gram staining and scoring of bacterial types by Nugent et al's (5) criteria. This technique correlates well with Amsel et al's clinical criteria $(5,6)$.

However, instead of sending an air-dried smear, clinicians commonly send a vaginal swab in transport medium to a laboratory for diagnosis of bacterial vaginosis. While this substitute for the direct smear of vaginal secretions is widely used in practice (4; personal communications - Ottawa Hospital, Ottawa, Ontario; McGill University Hospital Centre, Montreal, Quebec; Gamma Dynacare Medical Laboratories, Ottawa, Ontario), its accuracy and comparability to the direct smear have not been adequately studied. Furthermore, swabs in transport medium should be maintained at a controlled temperature and processed within $24 \mathrm{~h}$ of collection, which may be logistically difficult in rural areas or private clinics, particularly given our extremes of climate.

We sought to devise a new, simple, inexpensive sample collection method that would allow maximal flexibility in the transportation of specimens to a laboratory. One of the authors (MS) devised an alternate approach: take a sample of vaginal secretions with a cotton-tipped applicator, smear thinly onto a slide, and spray immediately with cytological fixative aerosol (Cytoprep, Fisher Scientific, Ontario). This contains isopropyl alcohol, propane and isobutane. This is the same fixative used for Pap smears. Preservation of the cytologically fixed slide is permanent. In a pilot study of 10 subjects, results of Nugent scoring obtained using vaginal smears sprayed with cytological fixative immediately after collection and Gram-stained one week later were compared with those from the standard preparation, an air-dried vaginal smear promptly heat-fixed and Gram-stained. The interval of one week was chosen as the longest time likely to be actually incurred in specimen transport. The preservation and staining of Gram-positive and -negative bacteria were excellent in both groups of slides, and diagnoses of bacterial vaginosis were concordant.

We now present the results of a larger, prospective study. We compare Nugent scores obtained using our 'user-friendly' method of a smear made directly from a vaginal swab and sprayed immediately with cytological fixative, sent to the laboratory seven days later for Gram staining; scores obtained using a vaginal swab placed in modified Amies clear transport medium and sent to the laboratory two days later; and scores from the reference air-dried smear of vaginal secretions, sent the same day to the laboratory for heat fixation and Gram staining.

\section{PATIENTS AND METHODS}

This anonymous, prospective study of specimen collection techniques for the diagnosis of bacterial vaginosis was carried out in 1999 and 2000. The Research Ethics Committee of The Ottawa Hospital approved the study protocol. Women attending a teaching hospital gynecology or colposcopy clinic or a public health 'healthy sexuality' clinic were invited to participate if they were having a gynecological speculum examination. Women were excluded if they had used a vaginal medication or douche within the previous week, if they had had already participated in the study and if they were younger than 18 years or not competent to give informed consent. No personal identifying or demographic characteristics were collected. To determine whether the same results were obtained with each alternate method as with the reference method, Cohen's kappa

\begin{tabular}{lccc}
$\begin{array}{l}\text { TABLE 1 } \\
\text { Distribution of Nugent scores of vaginal smears for } \mathbf{1 4 6} \\
\text { subjects, obtained by one technologist comparing transport } \\
\text { and fixation methods, with results for all three methods }\end{array}$ \\
\hline $\begin{array}{l}\text { Nugent score } \\
\text { mir-dried } \\
\text { method }\end{array}$ & $\begin{array}{c}\text { Amies medium } \\
\text { method }\end{array}$ & $\begin{array}{c}\text { Cytological } \\
\text { fixative method }\end{array}$ \\
\hline 0 & 85 & 82 & 88 \\
1 & 6 & 7 & 4 \\
2 & 1 & 2 & 1 \\
3 & 2 & 5 & 1 \\
4 & 8 & 8 & 8 \\
5 & 3 & 2 & 2 \\
6 & 0 & 0 & 2 \\
7 & 4 & 3 & 5 \\
8 & 22 & 26 & 20 \\
9 & 4 & 3 & 3 \\
10 & 11 & 8 & 12 \\
\hline
\end{tabular}

test for equivalence of matched pairs was used. A sample size of at least 125 subjects was required, assuming a 40\% prevalence of bacterial vaginosis in this population $(1,7)$, alpha $=0.05$ and $80 \%$ power to determine equivalence.

To ensure that each slide was assessed independently, each subject was assigned a subject identification (ID), which was linked to three unique random numbers, each corresponding to a specific sample method for that particular subject ID. Sealed packages containing the swabs, slides and transport medium for each subject were labelled with the subject ID only. The slides and the transport medium tube were labelled with their assigned, unique random numbers. During the examination with a nonlubricated speculum, three cotton-tipped swabs of upper vaginal secretions were taken. One swab was rolled onto a glass slide, which was sprayed immediately with cytological fixative. One swab was placed in modified Amies clear transport medium (Starplex Scientific, Canada). One swab was rolled onto a glass slide which was air-dried. The slides and transport medium tube were replaced in the study package, which was collected by the research assistant. The air-dried slide was delivered to the laboratory within $4 \mathrm{~h}$, the swab in transport medium was held at room temperature and delivered two days later and the slide sprayed with fixative was delivered seven days later. In the laboratory, a slide was prepared from the swab in transport medium. All slides were heat-fixed (because the technologist did not know whether a slide had been sprayed with fixative or air-dried) and then Gram-stained (with safranin counterstain). The Gramstained slides were prepared by a different laboratory technologist so that those technologists responsible for reading and Nugent scoring were blind to the sampling method. Each of two technologists independently assessed the quality (clarity and preservation of Gram-negative and -positive organisms) of each slide, noted the presence or absence of clue cells and ascertained the Nugent score (Table 1). No clinical information accompanied the specimens. If the Nugent score was seven or greater, it was categorized as positive, and bacterial vaginosis was diagnosed.

Bland-Altman plots (8) were used to display the agreement results between the air-dried specimens and each of the other two methods. These plots display the difference in scores between the methods against the mean score for each subject, thus highlighting any lack of agreement between the methods or relationship 


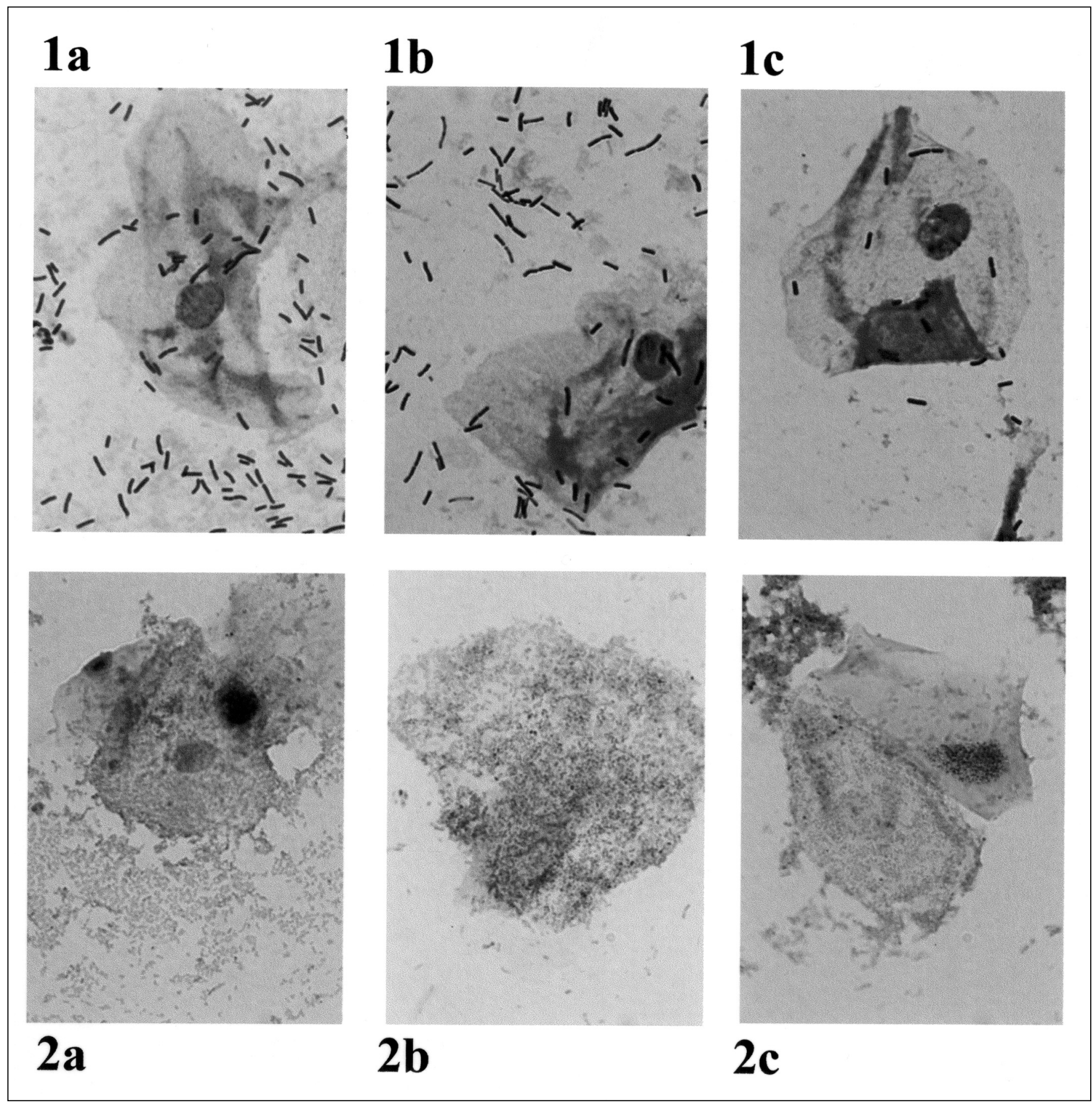

Figure 1) Gram-stained smears of vaginal secretions from two subjects. Subject 1 is negative and subject 2 is positive for bacterial vaginosis. a airdried smears, fixed and stained promptly; $\mathbf{b}$ smears made two days later from swab sent in Amies transport medium; $\mathbf{c}$ smears stained seven days later from slides sprayed promptly with cytological fixative (original magnification $\times 1000$ )

between measurement error and the score. Cohen's kappa test (9), an equivalence test for matched pairs of dichotomous results, was used to evaluate the number of paired samples giving the same (positive or negative) result for each of the two deferred transport methods relative to the air-dried, fixed specimens. The weighted Cohen's kappa test, which grants partial credit to scores that are close but not identical, was used to compare raw Nugent scores obtained by each deferred transport method to the air-dried method (9). Inter-rater reliability was also assessed. Cohen's kappa score is 0 when the results agree as often as expected by chance and 1 when there is complete agreement. Values of 0.41 to 0.60 indicate moderate agreement, 0.61 to 0.80 indicate substantial agreement and 0.81 to 1.0 indicate almost perfect agreement. Sensitivities and specificities with 95\% CIs were calculated for each transport and fixation method compared with the air-dried, fixed smears. Stata (StataCorp LP, USA) statistical software was used.

\section{RESULTS}

Data were available for the air-dried slide and at least one other specimen for 150 of the 152 subjects (six slides were broken 
TABLE 2

Comparison of diagnoses of bacterial vaginosis from slides prepared from swabs in Amies medium and from standard air-dried smears

\begin{tabular}{lcccc}
\hline & \multicolumn{2}{c}{ Air-dried smears } & Total \\
\hline \multirow{3}{*}{ Amies medium } & Positive & Negative & \\
& Positive & 37 & 3 & 40 \\
\multirow{2}{*}{ Total } & Negative & 4 & 103 & 107 \\
& & 41 & 106 & 147 \\
\hline
\end{tabular}

TABLE 3

Comparison of diagnoses of bacterial vaginosis from cytologically fixed smears and standard air-dried smears

\begin{tabular}{lcccc}
\hline & & \multicolumn{2}{c}{ Air-dried smears } & Total \\
\hline \multirow{2}{*}{ Cytological fixative } & Positive & Negative & \\
& Positive & 37 & 3 & 40 \\
\multirow{2}{*}{ Total } & Negative & 4 & 105 & 109 \\
& & 41 & 108 & 149 \\
\hline
\end{tabular}

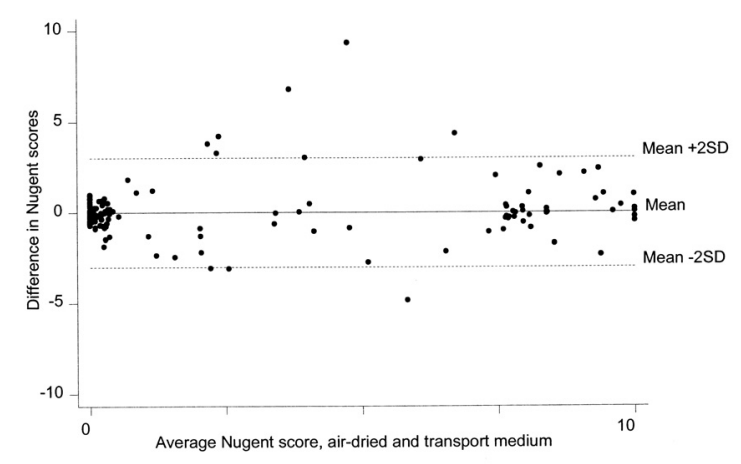

Figure 2) Bland-Altman plot comparing Nugent scores from slides airdried with slides prepared from swabs in Amies transport medium

or lost). Gram stain quality was rated as good in $92.6 \%$ of slides prepared from a swab in transport medium, $94.0 \%$ of slides with cytological fixative and $96.1 \%$ of air-dried slides. Gram stains prepared using each of the three methods from the same subject are shown in Figure 1.

Nugent scores obtained by the three techniques are shown in Table 1 for subjects with no missing slides. Of 146 subjects, $41(28.1 \%)$ had bacterial vaginosis diagnosed on the air-dried slide. When the diagnoses of bacterial vaginosis made from slides prepared from swabs in transport medium two days after collection were compared to those made from the reference air-dried specimens fixed the same day, 95.2\% were concordant, compared with $60.1 \%$ agreement expected by chance, kappa $=0.88$ (95\% CI 0.79 to 0.97) (Table 2). With respect to the air-dried standard, the 'transport medium' method had a sensitivity of $90.2 \%$ (95\% CI $85.3 \%$ to $95.1 \%$ ) and a specificity of $97.2 \%$ (95\% CI $94.5 \%$ to $99.9 \%$ ). When the diagnoses of bacterial vaginosis made from slides sprayed with cytological fixative and Gram-stained seven days later were compared with those made from the reference air-dried, promptly fixed specimens, $95.3 \%$ of scores were concordant, compared with an expected $60.4 \%$ agreement (kappa $=0.88$ [95\% CI 0.79 to

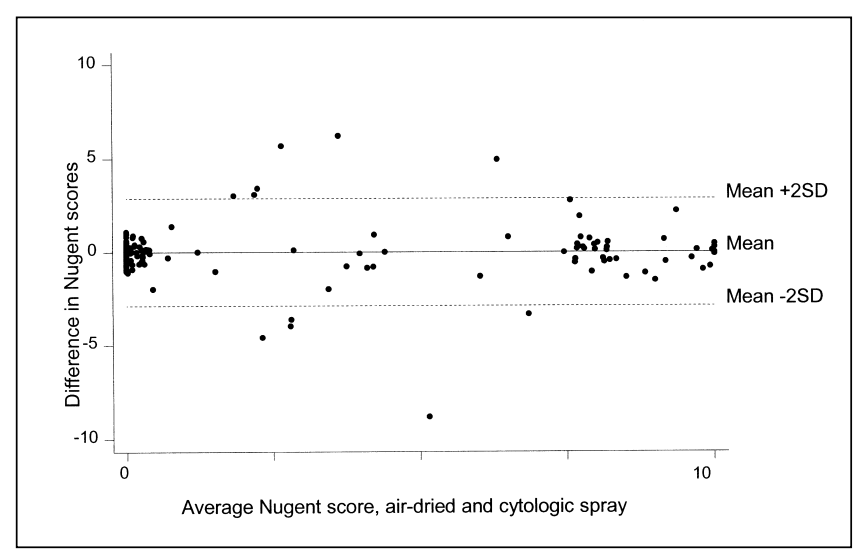

Figure 3) Bland-Altman plot comparing Nugent scores from slides airdried with slides sprayed with cytological fixative

\section{TABLE 4}

Inter-rater comparison for diagnosis of bacterial vaginosis (concordance $97.5 \%$, kappa for inter-rater reliability 0.94 , [95\% Cl 0.79 to 0.97$]$ )

\begin{tabular}{lcccc}
\hline & & \multicolumn{2}{c}{ Air-dried smears } & Total \\
\hline \multirow{2}{*}{ Technologist 1} & Positive & Negative & \\
& Positive & 110 & 10 & 120 \\
\multirow{2}{*}{ Total } & Negative & 1 & 327 & 328 \\
\hline
\end{tabular}

0.97]) (Table 3). With respect to the reference method, the 'cytospray' method had a sensitivity of 90.2\% (95\% CI 85.3\% to $95.1 \%)$ and a specificity of $97.2 \%(95 \%$ CI $94.5 \%$ to 99.9\%.)

We observed $93.5 \%$ agreement in raw Nugent scores between 'transport medium' and 'air-dried' samples (weighted kappa $=0.83, \mathrm{P}<0.0001$ ) (see Bland-Altman plot, Figure 2). The agreement on raw Nugent scores between 'cytospray' and 'air-dried' samples was 94.6\% (weighted kappa=0.86, $\mathrm{P}<0.0001$ ) (see Bland-Altman plot, Figure 3).

Scoring by the two technologists on all slides (three per subject) for diagnosis of bacterial vaginosis was highly concordant - the agreement was $97.5 \%$, compared with $61.7 \%$ expected by chance (kappa for inter-rater reliability $=0.94$ [95\% CI 0.79 to 0.97]) (Table 4). No significant difference was found in inter-rater reliability between methods.

In subjects diagnosed with bacterial vaginosis by Nugent's criteria, clue cells were noted more often on slides prepared from swabs sent in transport medium $(85.5 \%, 97.1 \%)$ or on slides with immediate cytological fixation (90.0\%, 94.6\%) than on air-dried, heat-fixed slides $(70.7 \%, 84.6 \%)$ by technologists 1 and 2, respectively.

\section{DISCUSSION}

The standard method for laboratory diagnosis of bacterial vaginosis is the Nugent et al's (5) semi-quantitative bacterial scoring of a smear of vaginal discharge, air-dried, fixed and Gramstained. Outside of regular working hours and the hospital context, it may be difficult to heat-fix specimens. Thompson (10) indicated that without rapid heat fixation, bacterial overgrowth may occur in a smear of vaginal secretions left for a long period of time. Although there is disagreement about the importance of 
delays in heat fixation (2), we could not locate any definitive published data. Sending a vaginal swab in transport medium for diagnosis of bacterial vaginosis is a common practice, but we found no published studies to validate using vaginal swabs instead of direct smears. Specimen dilution and delay in reaching the laboratory could potentially affect quantitation of bacterial types. Bartholomew and Mittwer (11) pointed out that "the age of the culture influences the Gram positivity of the culture", and we found no data examining the influence of residence time in transport medium on Gram positivity. We have now shown satisfactory sensitivity (90.2\%) and specificity $(97.2 \%)$ of Gram stains prepared from vaginal swabs compared with the direct smear. Even when swabs were processed $48 \mathrm{~h}$ after collection, bacterial morphotypes and quantitation were well preserved.

However, with the extremes of the Canadian climate, the trend toward regionalization of microbiology laboratories, and in rural areas, maintaining swabs at $4^{\circ} \mathrm{C}$ to 'room' temperature for delivery to the lab within $24 \mathrm{~h}$ may be costly. Indeed, the cost of a swab and transport medium is greater than that of a slide, and is completely avoidable. Our method of cytological fixation of a fresh vaginal smear eliminates the need for prompt specimen transport at a controlled temperature. The quality of slides prepared in this way was equivalent to that of air-dried smears; inter-rater reliability was similar. The sensitivity (90.2\%) and specificity (97.2\%) for diagnosis of bacterial vaginosis by Nugent's criteria were excellent. Preservation of clue cells was superior, which is not surprising, because air-drying affects cellular detail. This simple method uses materials available wherever pelvic examinations are completed. Because the specimen is already fixed, the vaginal smear sprayed immediately with cytological fixative has advantages over both the air-dried smear and the swab in transport medium. It is of particular

\section{REFERENCES}

1. Amsel R, Totten PA, Spiegel CA, et al. Nonspecific vaginitis: Diagnostic criteria and microbial and epidemiologic associations. Am J Med 1983;74:14-22.

2. Hillier SL, Holmes KK. Bacterial vaginosis. In: Holmes KK, Mardh P-A, Sparling PF, et al, eds. Sexually Transmitted Diseases. New York: McGraw Hill, 1999.

3. Schwebke JR. Diagnostic methods for bacterial vaginosis. Int J Gynaecol Obstet 1999;67(Suppl 1):S21-3.

4. Mazzulli T, Simor AE, Low DE. Reproducibility of interpretation of gram-stained vaginal smears for the diagnosis of bacterial vaginosis. J Clin Microb 1990;28:1506-8.

5. Nugent RP, Krohn MA, Hillier SL. Reliability of diagnosing bacterial vagnosis is improved by a standardized method of gram stain interpretation. J Clin Microb 1991;29:297-301.

6. Schwebke JR, Hillier SL, Sobel JD, McGregor JA, Sweet RL. Validity of the vaginal gram stain for the diagnosis of bacterial vaginosis. Obstet Gynecol 1996;88:573-6.

7. Davis JD, Connor EE, Clark P, Wilkinson EJ, Duff P. Correlation between cervical cytologic results and Gram stain value to permit quality control assessment at a reference laboratory, or centralized, consistent handling and interpretation for research into the role of bacterial vaginosis in premature birth and infectious complications in obstetrics and gynecology. The excellent preservation of bacterial morphotypes and Gramstaining characteristics in the cytologically fixed specimens suggests that this approach could be used for other clinical samples in which delayed Gram staining may be desirable.

Other approaches to the diagnosis of bacterial vaginosis have been investigated. For example, use of the cervical Pap smear instead of a vaginal specimen to diagnose bacterial vaginosis has been shown to be insensitive $(7,12)$. Papanicolaou staining of a vaginal (rather than cervical) smear has better sensitivity for bacterial vaginosis (13). On the other hand, with Papanicolaou staining, the Gram reaction of bacteria cannot be assessed, so Nugent scoring is not possible. Oligonucleotide probes for Gardnerella species bacteria have been developed, but they are costly, and the presence of Gardnerella species is not diagnostic of bacterial vaginosis. Nugent scoring of a Gram stain, therefore, remains the microbiological procedure of choice. We have confirmed the equivalence of results obtained from vaginal swabs sent in transport medium or from cytologically fixed vaginal smears to results from air-dried, heat-fixed vaginal smears for the diagnosis of bacterial vaginosis by Nugent scoring.

ACKNOWLEDGEMENTS: This research was supported by the Canadian Foundation for Women's Health. We are indebted to microbiology technologists W Woods and D Edgar; Drs M Gordon, M Al-Maktoum and K Ramotar; as well as the staff of the Healthy Sexuality Clinic and the Ottawa Hospital Gynaecology Clinic.

as diagnostic tests for bacterial vaginosis. Am J Obstet Gynecol 1997;177:532-5.

8. Bland JM, Altman DG. Statistical methods for assessing agreement between two methods of clinical measurement. Lancet 1986;8:307-10.

9. Cohen J. Weighted kappa: Nominal scale agreement with provision for scaled disagreement or partial credit. Psychol Bull 1968;70:213-20.

10. Thompson DW. Adequate Pap Smears: A Guide for Sampling Techniques in Screening for Abnormalities of the Uterine Cervix. Toronto: Ontario Medical Association, 1996.

11. Bartholomew JW, Mittwer T. The Gram stain. Bacteriol Rev 1952;16:1-29.

12. Lamont RF, Hudson EA, Hay PE, et al. A comparison of the use of Papanicolaou-stained cervical cytological smears with Gram-stained vaginal smears for the diagnosis of bacterial vaginosis in early pregnancy. Int J STD AIDS 1999;10:93-7.

13. Giacomini G, Calcinai A, Moretti D, Cristofani R. Accuracy of cervical/vaginal cytology in the diagnosis of bacterial vaginosis. Sex Transm Dis 1998;25:24-7. 